\title{
DYNAMICS OF ROTATING STELLAR SYSTEMS: COLLAPSE AND VIOLENT RELAXATION*
}

\author{
J. RICHARD GOTT III \\ California Institute of Technology, Pasadena, Calif., U.S.A.
}

\begin{abstract}
Axisymmetric systems, having differing amounts of initial angular momentum and containing 2000 stars (mass points) each, are allowed to collapse under their own gravitational attraction. Collapse and violent relaxation are found to lead to the formation of equilibrium structures after only a few free-fall times. The systems contain a sufficient number of mass points so as to be effectively collisionless and the equilibrium structures are consistent with accurate satisfaction of the stationary collisionless Boltzmann equation. Rotating equilibrium models produced in this way resemble elliptical galaxies, with elliptical isophotes. With cosmologically reasonable amounts of initial angular momentum, E0 to E4.5 galaxies can be produced. The equilibrium models show rotation curves in general agreement with that expected from violent relaxation theory. In particular the models show a central region with solid-body rotation. Further out, the systems become differentially rotating. When infall effects are included one can produce models with extended envelopes in good agreement with observed elliptical galaxies. It is suggested that the crucial factor in determining whether an elliptical or a spiral galaxy is formed is whether or not star formation is complete by the time the proto-galaxy reaches its point of maximum collapse.
\end{abstract}

I shall discuss several dynamical models I have made for the formation of elliptical galaxies, models which cover the collapse and violent relaxation phase leading to the formation of the equilibrium galaxy. The approximation of an axisymmetric gravitational field is utilized to simplify the $N$-body calculation and thus allow treatment of many more stars than would otherwise be possible. In particular, sufficient stars may be treated to achieve a system which is effectively collisionless.

The procedure can be outlined as follows. Our rotating systems are axisymmetric and contain 2000 mass points (henceforth called stars) of equal mass. To derive the gravitational potential $\Phi$ due to these stars, we divide space into a network of toroidal cells centred on the rotation axis of the system and having approximately square cross-sections. The mass within each cell is assumed to be concentrated on the central ring of the cell and the values of the potential are then calculated on a series of rings forming the corners of the toroidal cells. The potential within each cell is found by linear interpolation from the values at the cell's four corner rings. At the end of each time step, this approximate gravitational potential is used to find the force on each star, the stellar trajectories are then advanced and the process is repeated. The network of toroidal cells is set up by constructing a spherical coordinate system $(r, \theta, \phi)$ centred on the galaxy and whose axis $\theta=0$ is the rotation axis of the galaxy. At the origin we establish a small reflecting sphere of radius $R_{\mathrm{s}}$. Let $\alpha=\pi / 18$ or an angle of $10^{\circ}$. Then the cell $C_{i j}$ contains all points with coordinates in the ranges:

$$
(i-1) \alpha<\ln \left(r / R_{\mathrm{s}}\right)<i \alpha, \quad(j-1) \alpha<\theta<j \alpha, \quad 0 \leqslant \phi<2 \pi .
$$

\footnotetext{
* Supported in part by the National Science Foundation [GP-36687X, GP-28027].
} 
Each cell has an angular diameter of $10^{\circ}$ in $\theta$ and an outside radius approximately $19 \%$ larger than the inside radius. This gives each cell an approximately square cross section. In our calculations we use 24 layers of 18 cells each: $C_{i j}(i=1,24 ; j=1,18)$. These cells cover a spherical volume of radius $\exp (24 \alpha) R_{\mathrm{s}} \simeq 64 R_{\mathrm{s}}$. Stars passing beyond this outer radius are assumed to escape; stars hitting the inner reflecting sphere are reflected elastically from its surface. Calculation of the potentials and advance of the trajectories of the 2000 stars requires approximately 79000 operations per elementary time step compared with $3 N^{2}=12 \times 10^{6}$ operations per time step required for an $N$-body calculation with $N=2000$.

Our models are started at the cosmological epoch of maximum expansion of the protogalaxy. We then follow the collapse and violent relaxation for four models, I, II, III, IV with increasing amounts of initial angular momentum. Each of the models begins as a system of 2000 stars distributed randomly so as to produce a uniformly dense sphere of stars of total mass $M_{\mathrm{gal}}$ and radius $R_{0} . R_{0}$ thus represents the maximum radius of expansion of the protogalaxy. If such a uniform sphere of stars were set in uniform (solid-body) rotation with angular rate $\Omega_{0}=\left(G M_{\mathrm{gal}} / R_{0}^{3}\right)^{1 / 2}$, then instantaneously it would be holding itself up against gravity in its equatorial plane. The stars in each galaxy are given systematic velocities $V_{\phi}$ so that the initial state is one of uniform rotation with angular rate $\Omega_{i}$, where for Model I, $\Omega_{i}=0$ (no rotation); for Model II, $\Omega_{i}=\left(\frac{1}{4}\right)^{1 / 2} \Omega_{0}$; for Model III, $\Omega_{i}=\left(\frac{1}{2}\right)^{1 / 2} \Omega_{0}$; and for Model IV, $\Omega_{i}=$ $=\left(\frac{3}{4}\right)^{1 / 2} \Omega_{0}$. Superimposed upon this systematic rotation, the stars are given small random velocities with a Maxwellian distribution so that $\left\langle V_{r}^{2}\right\rangle^{1 / 2}=\left\langle V_{\theta}^{2}\right\rangle^{1 / 2}=$ $=\left\langle\left(V_{\phi}-\left\langle V_{\phi}\right\rangle\right)^{2}\right\rangle^{1 / 2}$. Initially $\left\langle V_{\mathrm{r}}\right\rangle=\left\langle V_{\theta}\right\rangle=0$. In Models I, II, and III the random velocities were chosen so that the initial virial coefficient for the random kinetic energy, $\left|2 T_{\text {random }} / W\right|$, was equal to one half, where $W=-\left(3 G M_{\mathrm{gal}}^{2} / 5 R_{0}\right)$. In Model IV the energy in the random velocities was reduced from that above by a factor of 2.

Let us now also define some useful quantities. Let $\varrho_{0}=\left(3 M_{\mathrm{gal}} / 4 \pi R_{0}^{3}\right)$ be the standard density and $V_{0}=\left(3 G M_{\mathrm{gal}} / 5 R_{0}\right)^{1 / 2}$ be the standard virial velocity. Let $E_{\mathrm{b}}=$ $=-(T+W)$ be the binding energy for each model. Define for each model the energy radius $R_{\mathrm{E}}=\left(3 G M_{\mathrm{gal}}^{2} / 10 E_{\mathrm{b}}\right)$ which is the characteristic radius of a system in virial equilibrium with binding energy $E_{\mathrm{b}}$. Define a characteristic oscillation time $T_{\mathrm{c}}=2 \pi\left(R_{E}^{3} / G M_{\mathrm{gal}}\right)^{1 / 2} . T_{\mathrm{c}}$ is twice the free-fall time of a galaxy of mass $M_{\mathrm{gal}}$, binding energy $E_{\mathrm{b}}$, and initial radius $r_{\max }=2 R_{\mathrm{E}}$ (see Spitzer, 1968). The age of the Universe when $r=r_{\max }$ is thus $\left(\frac{1}{2}\right) T_{\mathrm{c}}$; the age of the Universe at the end of the collapse is $T_{\mathrm{c}}$. The times in each model are measured in terms of $T_{\mathrm{c}}$ for that model.

Models I-IV possess reasonable amounts of cosmological rotation such as might be obtained through tidal interactions with neighbouring protogalaxies (cf. Peebles, 1971). These rotating spheres of stars are then allowed to collapse under their own gravitational force. During the collapse phase the potential oscillates violently, producing the Lynden-Bell violent relaxation, and one finds for these rotating systems, just as for spherical systems, that they reach relaxed equilibrium configurations after only a couple of free-fall times.

Let us examine our expectations for the equilibrium models. Consider a non-rotat- 
ing sphere of stars with no initial kinetic energy and a radius of $R_{0}$. Then $-E_{\mathrm{b}}=T+W$ with $W=-\left(3 G M_{\mathrm{gal}}^{2} / 5 R_{0}\right)=-E_{\mathrm{b}}$ and $T=0$. Let it collapse and undergo violent relaxation. When virial equilibrium is reached, $2 T+W=0$ and $-E_{\mathrm{b}}=T+W$ so $W=-2 E_{\mathrm{b}}$, or twice its original value, and the equilibrium system will be spherical with a characteristic radius of $\sim\left(\frac{1}{2}\right) R_{0}$. Now consider a rotating galaxy of radius $R_{0}$ and $\Omega=\Omega_{0}$ so that it is initially holding itself up against gravity in the equatorial plane. This is surely an upper limit on the amount of rotation we would expect cosmologically. Let this galaxy collapse. It continues holding itself up in the equatorial plane, leaving $r \sim R_{0}$ in that direction. Rotation does not inhibit collapse perpendicular to the plane, so to first approximation the collapse proceeds perpendicularly as in the

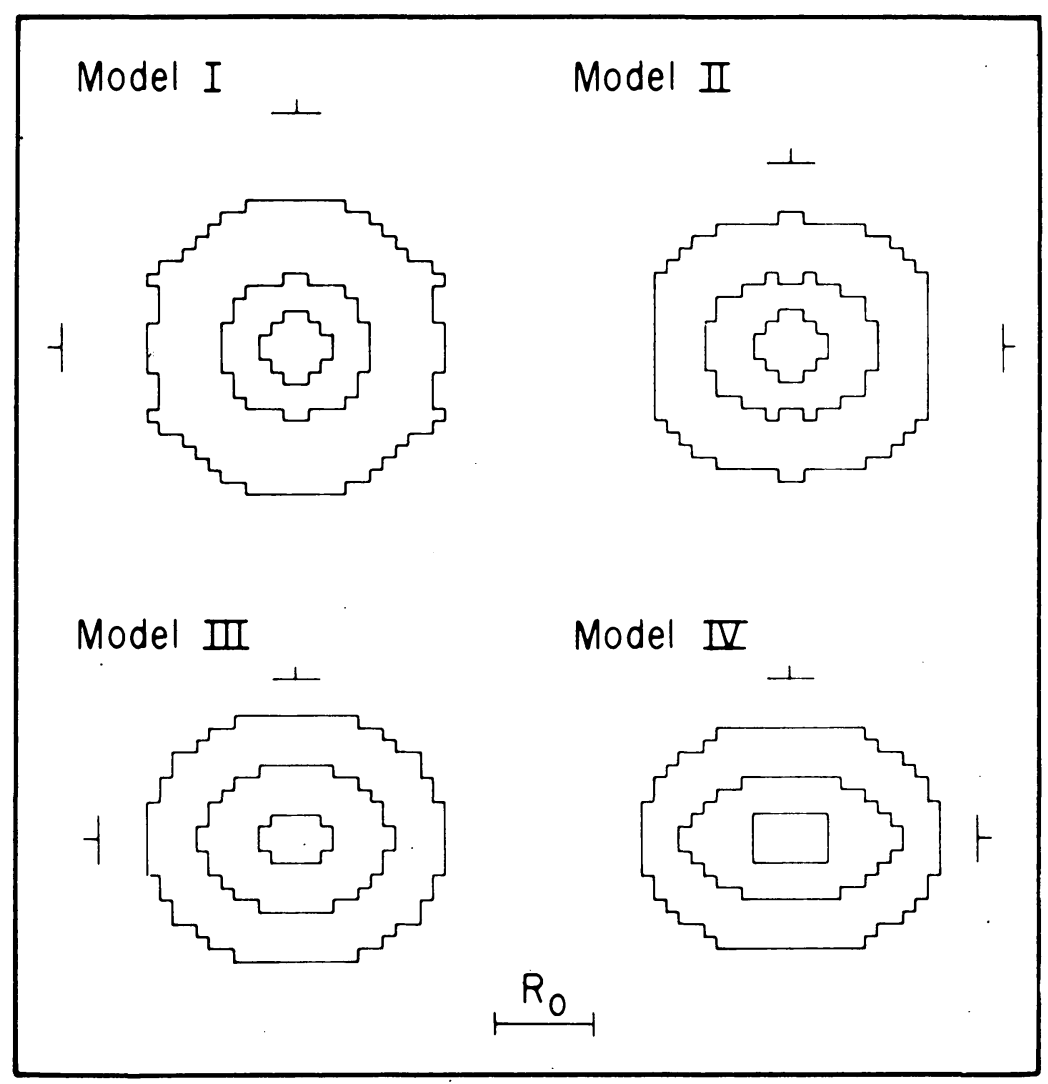

Fig. 1. Isophotes for the equilibrium galaxies. The diagram represents the appearance of these galaxies to a distant observer whose line of sight lies in their equatorial plane. The rotation axis of each galaxy is vertical and the equatorial plane is horizontal. The innermost isophote represents a projected surface density of $I_{0}=0.8 \mathrm{Mgal}_{\mathrm{gal}} / R_{0}{ }^{2}$. The next two isophotes represent intensities of $0.1 I_{0}$ and $0.01 I_{0}$, while the tick marks indicate the extent of the $0.001 I_{0}$ isophote. The isophotes are computed from a grid in one quadrant only, thereby giving the diagrams a four-fold symmetry. The scale is given at the bottom: all four galaxies were initially spheres of radius $R_{0}$ before undergoing collapse and violent relaxation. Model I had no initial rotation and is therefore spherical (E0). Models II-IV had progressively larger amounts of initial rotation, and have produced progressively more elliptical equilibrium galaxies (E1.5, E2.5, E4.5). 
spherical case to give $r \sim\left(\frac{1}{2}\right) R_{0}$. These approximations indicate an equilibrium galaxy with a 2 to 1 axial ratio (i.e., an E5 galaxy). Thus, with the maximum reasonable amount of initial rotation, we get an equilibrium galaxy with a flattening of at most E5. Only in dissipative systems with gas present can we produce flat systems such as spiral galaxies.

In Figure 1 are shown the isophotes for the equilibrium galaxies. They represent (as do later data) time averages over the period $\left(\frac{3}{2}\right) T_{\mathrm{c}}<t<\left(\frac{11}{2}\right) T_{\mathrm{c}}$. The equilibrium models are indeed elliptical galaxies, with isophotes that are elliptical in shape, and Models I-IV correspond approximately to E0, E1.5, E2.5, E4.5 galaxies, respectively. Our general expectations are confirmed: Model I, with no rotation, is quite spherical and the models with greater initial angular momentum are more flattened but not flatter than E5. Peebles' (1971) favoured value for angular momentum gained by gravitational interactions would give nearly spherical galaxies (Model II), but his upper bound would allow production of E3 and E4 galaxies. Earlier in this conference Dr de Vaucouleurs summarized (p. 1) the observational data on the intrinsic flattening in elliptical galaxies. The results show elliptical galaxies to have an intrinsic

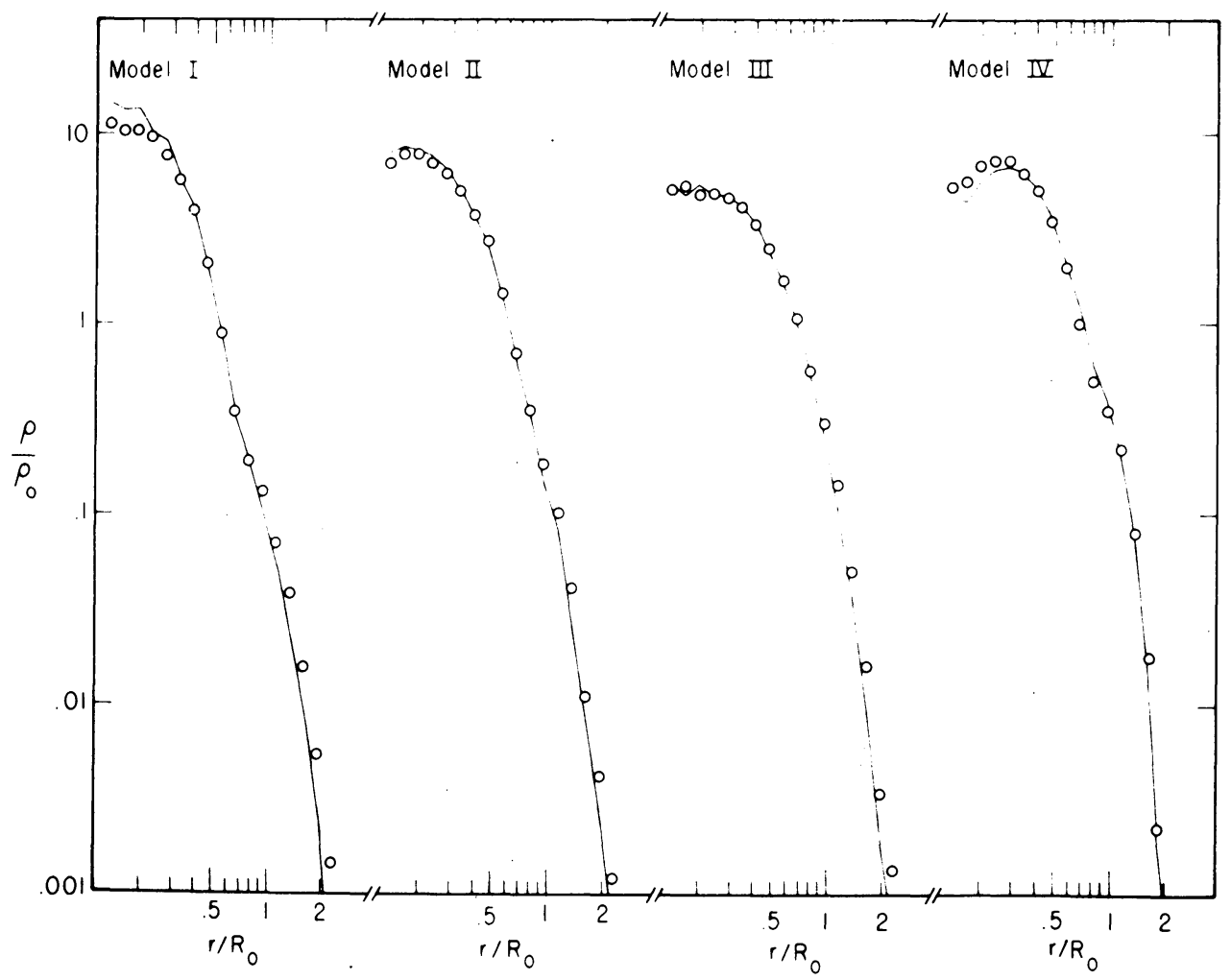

Fig. 2. Stellar density in the galactic plane as a function of radius for the equilibrium models. The open circles represent the actual runs of density with radius while the solid line represents that expected from satisfaction of the stationary collisionless Boltzmann equation. $Q_{0}=3 M_{\mathrm{gal}} / 4 \pi R_{0}{ }^{3}$ is a standard pre-collapse density which is the same for all four models. 
flattening between E0 and E5 with only a few E6's and no E7 galaxies, in qualitative agreement with what we predict for dissipationless collapse models.

In Figure 2 the stellar density in the galactic plane as a function of distance from the centre is plotted for each model. The data points are densities from successive cells lying on the galactic plane. The solid line shows the density expected from satisfaction of the stationary, collisionless Boltzmann equation:

$$
\begin{aligned}
-\frac{\partial \Phi}{\partial r}=- & \frac{\left\langle V_{\phi}\right\rangle^{2}}{r}+\left\langle V_{r}^{2}\right\rangle\left[\frac{\partial \ln \varrho}{\partial r}+\frac{\partial \ln \left\langle V_{r}^{2}\right\rangle}{\partial r}+1\left(1-\frac{\left\langle V_{\theta}^{2}\right\rangle}{\left\langle V_{r}^{2}\right\rangle}\right)+\right. \\
& \left.\frac{1}{r}\left(1-\frac{\left\langle\left(V_{\phi}-\left\langle V_{\phi}\right\rangle\right)^{2}\right\rangle}{\left\langle V_{r}^{2}\right\rangle}\right)\right] .
\end{aligned}
$$

To compute the solid lines in Figure 2 we use our approximate potential and evaluate all velocities and velocity dispersions (within cells) as a function of radius with derivatives replaced by differences. We solve for $\ln \varrho$ as a function of $r$, and normalize. Very good agreement is found between the actual runs of density and those predicted from satisfaction of the collisionless Boltzmann equation. This demonstrates that the equilibrium models are stationary and essentially collisionless.

Rotation curves for the equilibrium models are presented in Figure 3. Significantly, all three rotating models show a central region of solid-body rotation. Lynden-Bell (1967) has shown that the statistically most probable distribution function for a rotating system is one of solid-body rotation with isothermal Maxwellian residual velocities. Thus our models show that the violent relaxation is efficient enough to produce this 'most probable' solid-body rotation at least in a central region. Also plotted in Figure 3 are rotation curves calculated by Lynden-Bell (1967), taking into account partial relaxation effects. Our models show very good general agreement with Lynden-Bell's theoretical prediction. Also presented for comparison are rotation curves from a self-consistent model by Prendergast and Tomer (1970) which assumes a distribution function with Maxwellian residual velocities and a tidal energy cut-off. The model's behaviour is not in agreement with that predicted by Prendergast and Tomer (1970) whose sharp tidal cut-off artificially drags their rotation curves down to zero at a certain radius.

Figure 4 shows the velocity dispersions for Models I, II, and IV. In Model I which is non-rotating we see a core region where the velocity distribution is isotropic and isothermal. This is to be expected due to the violent relaxation. Outside the core we find a halo where the velocity dispersion is largest in the radial direction. In the rotating models, isotropy of the random velocities in the centre is not achieved nearly as well as in the spherical system, particularly in the equatorial plane where the velocity dispersion perpendicular to the plane is the largest component. This is not unreasonable physically for a system which has collapsed primarily in the direction perpendicular to the plane. The departures from isotropy in the centre become progressively larger as we go toward more rapidly rotating systems. A more detailed presentation of these model results appears in Gott (1973). 


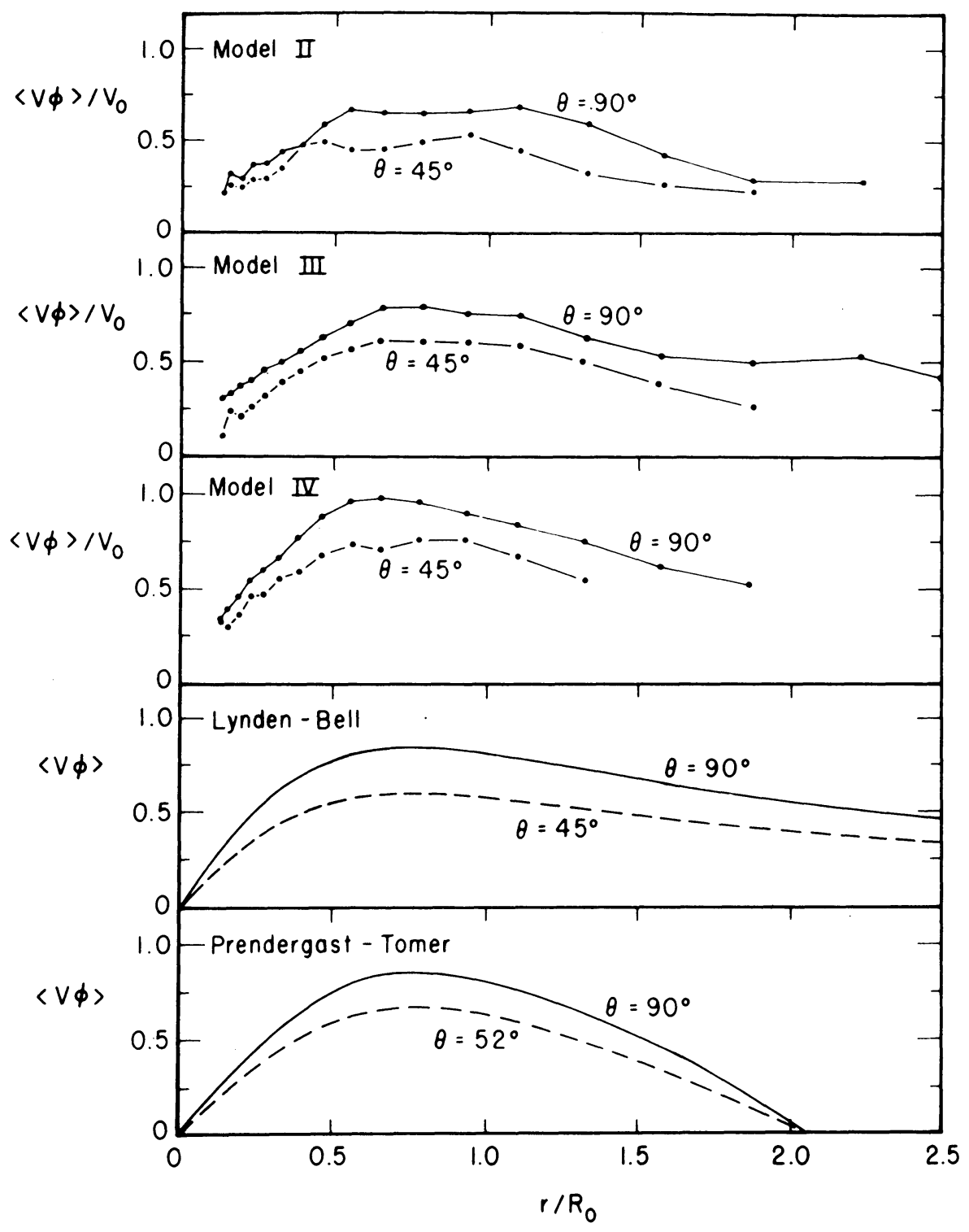

Fig. 3. Rotation curves. $\theta=90^{\circ}$ is in the equatorial plane, $\theta=45^{\circ}$ is along a line halfway between the equatorial plane and the axis of rotation. Results from the three rotating models are presented. They show approximate solid-body rotation in the centre and differential rotation further out. For comparison are two normalized theoretical curves: Lynden-Bell (violent relaxation and estimates of partial relaxation effects) and Prendergast and Tomer (self-consistent equilibrium models with Maxwellian random velocities and a tidal energy cut-off). 
The models we have been considering are quite simple ones. They are produced by the collapse of an isolated sphere of stars. A truly realistic model should include not only the protogalaxy itself, but should consider the protogalaxy in a complete cosmological setting. In addition to the protogalaxy itself, there will be outlying, still expanding material which is cosmologically bound to it and which will suffer infall into the protogalaxy after the protogalaxy has completed its collapse (cf. Gott and Gunn, 1971). This infall material will give the galaxy a less tightly bound, extended envelope. I have recently completed a series of models which take into account these infall effects and for which the angular momentum of the protogalaxy is derived directly from a tidal interaction with a neighbouring protogalaxy. The results of these calculations are, I think, quite promising. They show that with infall one can produce ex-

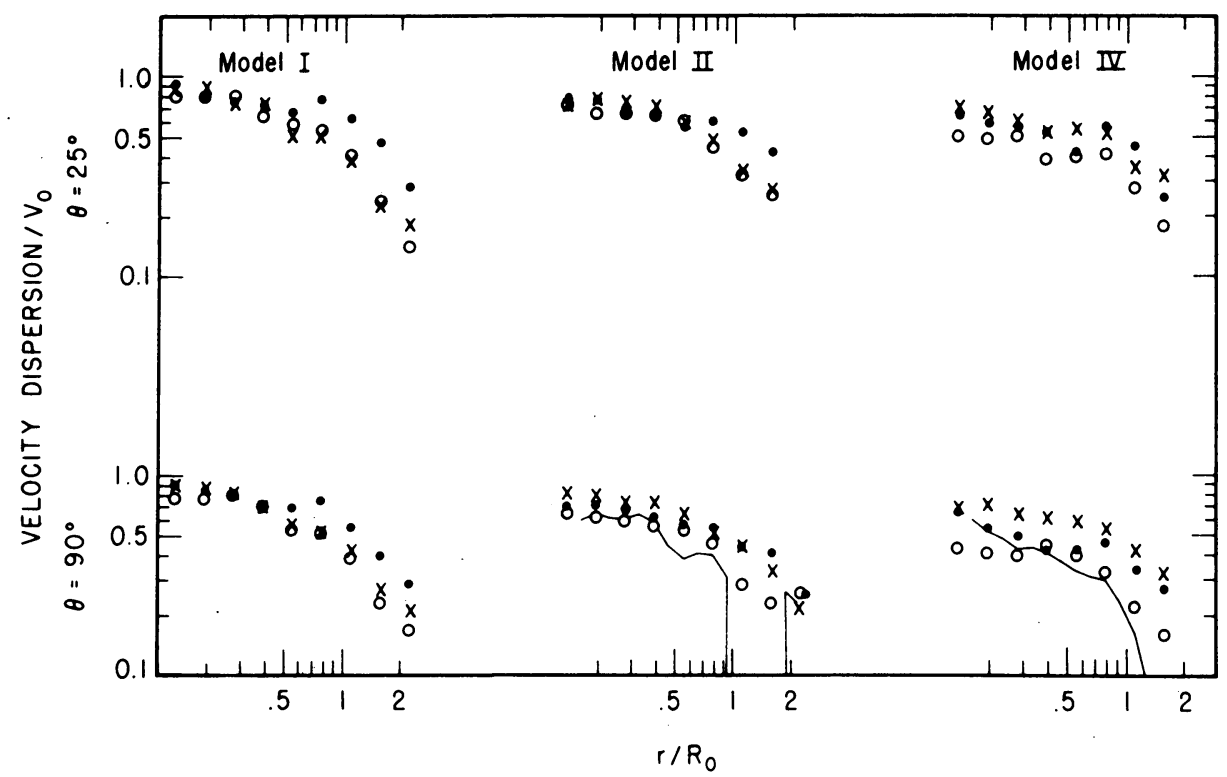

Fig. 4. Velocity dispersion as a function of radius from the centre. $\theta=90^{\circ}$ is in the galactic plane, $\theta=25^{\circ}$ is along a line making an angle of $25^{\circ}$ with the rotation axis. A spherical coordinate system $(r, \theta, \phi)$ is used.

$$
\begin{aligned}
& O=\left\langle V_{r}^{2}\right\rangle^{1 / 2}, \\
& \times=\left\langle V_{\theta^{2}}\right\rangle^{1 / 2}, \\
& O=\left\langle\left(V_{\phi}-\left\langle V_{\phi}\right\rangle\right)^{2}\right\rangle^{1 / 2} .
\end{aligned}
$$

$V_{0}=\left(3 G M_{\mathrm{gal}} / 5 R_{0}\right)^{1 / 2}$ is a standard velocity which is the same for all systems. Since Model $\mathrm{I}$ is spherical, its curves for $\theta=90^{\circ}$ and $\theta=25^{\circ}$ are similar. It has a very isotropic velocity distribution near the centre; far from the centre the halo stars dominate with their preferentially radial dispersions. A similar but smaller halo effect can be seen in Model II $\left(\theta=25^{\circ}\right)$. The more rapid the rotation, the less isotropic the velocity distributions become. Better isotropy is achieved near the rotation axis than in the galactic plane. For the two rotating models, the solid curve represents the expected value of $\left\langle\left(V_{\phi}-\left\langle V_{\phi}\right\rangle\right)^{2}\right\rangle^{1 / 2}$ (given $\left\langle V_{r}^{2}\right\rangle^{1 / 2}$ and the rotation curve) under the assumption of a Schwarzschild velocity distribution everywhere and satisfaction of the stationary collisionless Boltzmann equation. In Model II, $\left\langle V_{\phi}\right\rangle$ falls off slightly faster than $r^{-1}$ for a short stretch so the solid curve drops to zero there. Results for Model III are intermediate between Models II and IV and are not presented. 
tended envelopes like those observed in actual elliptical galaxies. Also it seems possible to produce model galaxies with isophotal eccentricities in their envelopes similar to those observed in real galaxies.

It should be emphasized that all of these models have no gaseous dissipation in them. That is, they represent models in which star formation is essentially completed by the time the galaxy reaches its point of maximum collapse. Such early star formation might take place in the primeval globular clusters, as suggested by Peebles and Dicke (1968).

There are several reasons to think that elliptical galaxies might be formed in just this manner. First, as I have mentioned, the observed eccentricities of elliptical galaxies are in good qualitative agreement with those predicted from a dissipationless collapse. Second, in elliptical galaxies there is an observed tendency for the isophotes to become rounder as one approaches the centre of the galaxy. (Note, for example, behaviour of this sort in Models II and IV.) If gaseous dissipation were important, we would expect the inner isophotes of the galaxy always to be flatter than the outer ones, which is not the case. Finally, the Lynden-Bell violent relaxation operating in a free-fall collapse without dissipation offers an attractive way to produce the relaxed, ellipsoidal configurations of stars we observe in elliptical galaxies.

I have made other models of collapsing protogalaxies which include both gas and stars. These give the expected results. A rotating sphere composed of stars and gas clouds (having inelastic collisions) was allowed to collapse, with the gas clouds slowly being turned into stars as the collapse proceeded. During the collapse phase, the stars and gas moved together as a unit and the sphere proceeded through a series of flattened ellipsoids until it became a flat pancake as the material reached the plane.

Lynden-Bell (1967) has shown that a uniformly rotating, uniform density fluid sphere collapses homologously through a series of uniformly rotating MacLaurin ellipsoid configurations. The gas clouds do not dissipate any significant amount of energy during the homologous collapse, because each cloud sees clouds in its neighbourhood moving with similar velocity to itself. When the material reaches the plane, this situation changes: the stars pass through the plane on their free trajectories, undergo violent relaxation, and form a relaxed spheroidal component similar to that seen in the elliptical galaxy models we have discussed. The gas, however, dissipates its energy quickly in the vertical direction to form a thin disk. The critical factor is the amount of gas left at the time of maximum collapse. Whatever gas is left at this time will dissipate its energy and form a disk. If some of this gas subsequently turns into stars, the new stars will also be confined to the disk.

Thus, we have a reasonable scenario for the formation of elliptical and spiral galaxies. In elliptical galaxies the star formation is essentially complete by the time of maximum collapse and a spheroidal galaxy is produced. If star formation is not completed by the time of maximum collapse and substantial amounts of gas are left over, then gaseous dissipation is important and a flat, disk-like spiral galaxy is produced. In this conference (e.g. p. 130) it has been emphasized that spiral galaxies show both disk and spheroidal components. We can find a continuous sequence from ellipticals 
with no disk, to spirals with a spheroidal nuclear bulge, to spirals with no observable spheroidal component. The value of $M_{\text {disk }} / M_{\text {spheroidal }}$ varies smoothly along the sequence. With the present model it is easy to see how such a sequence would originate: by varying the initial star formation rate we could have any desired fraction of leftover gas at the time of maximum collapse and therefore produce any given ratio of $M_{\text {disk }} / M_{\text {spheroidal }}$.

In summary then, we have presented dynamical models for the formation of elliptical galaxies. The equilibrium elliptical galaxy is produced by a simple free-fall collapse and violent relaxation process. The present picture offers a natural mechanism for forming elliptical and spiral galaxies. The early star formation rate is seen as the key factor in determining which type of galaxy is produced.

\section{References}

Gott, J. R., III: 1973, Astrophys. J. 186, 481.

Gott, J. R., III and Gunn, J. E.: 1971, Astrophys. J. Letters 169, L13.

Lynden-Bell, D.: 1967, Monthly Notices Roy. Astron. Soc. 136, 101.

Peebles, P. J. E.: 1971, Astron. Astrophys. 11, 377.

Peebles, P. J. E. and Dicke, R. H.: 1968, Astrophys. J. 154, 891.

Prendergast, K. H. and Tomer, E.: 1970, Astron. J. 75, 674.

Spitzer, L., Jr.: 1968, Diffuse Matter in Space, Wiley and Sons, New York.

\section{DISCUSSION}

Contopoulos: Did you take into account encounters between neighbouring stars?

Gott: Calculations show that placing the stars in these toroidal cells has the effect of making the stars gravitate as if they were rings, with a cut-off in their gravitational attraction if they approach closer than half the cell-width. In other words, once two stars are within the same toroidal cell they exert no more gravitational attraction on each other. The toroidal cells each have a nearly square cross-section with a cross-sectional width and breadth approximately $19 \%$ as large as their radius. The volume of space into which the galaxy is placed is divided into 432 such toroidal cells. Encounters between stars (i.e. encounters between rings) are thus handled in a natural fashion. The systems contain a sufficient number of stars so that the effects of two-body encounters are insignificant and the systems can be regarded as effectively collisionless.

Miller: Similar calculations have been run by plasma physicists for studies of plasma confinement, using about 120000 pseudo-particles in the form of rings in cylindrical coordinates. These calculations have been done by Morse et al. at Los Alamos (in B. Alder et al., Methods in Computational Physics, Academic Press, N.Y., 9, 213), by Killeen et al. at Livermore (J. Comput. Phys. 11, 360, 1973), and by the group at Culham. For ellipticals, such ring models should be satisfactory.

Mark: Can you please repeat again your evidence that the evolution is collisionless? You do not have very many rings.

Gott: First, theoretical estimates were made of the effective two-body relaxation time for this particular numerical scheme with $N=2000$. These results indicated that $T_{\mathrm{R}} \sim 120 T_{\mathrm{c}}$, where $T_{\mathrm{c}}$ is the characteristic orbital time of the systems. We study the models only out to a time $t=5.5 T_{\mathrm{c}}$, so the systems are effectively collisionless over this time-scale. After the systems reached their equilibrium configurations, the individual stellar energies were plotted to watch the random walk in individual stellar energy due to two-body encounters. Values of $\left\langle(\Delta E)^{2} / E^{2}\right\rangle$ for stars over the observed period yielded values of $T_{\mathrm{R}} \sim 60 T_{\mathrm{c}}$, consistent with the theoretical estimates and indicating an effectively collisionless system. Additional checks included the fact that no deepening of the central potential well (or change in any of the equilibrium model parameters) was observed between $t=(3 / 2) T_{\mathrm{c}}$ and $t=(11 / 2) T_{\mathrm{e}}$. Finally, there is the check that the equilibrium models accurately satisfy the stationary collisionless Boltzmann equation as indicated in Figure 2. 
Carrick: The rotating models of Prendergast and Tomer (Astron. J. 75, 674, 1970) have velocity dispersions in each of three dimensions equal near the centre, since this region is nearly isothermal. In your models, for stronger rotation, the three are significantly different, even close to the centre. Is this a statistical effect?

Gott: No. The predominance of the velocity dispersion perpendicular to the galactic plane for locations in the plane is shown consistently for a range of radii in each of the three rotating models. The effect seems physically reasonable for systems that have suffered their main collapse perpendicular to the plane. It becomes progressively more pronounced as one goes toward the more rapidly rotating models for which the violent relaxation is simply not effective enough to produce isotropic velocity dispersions.

Tifft: Some galaxies in the Coma cluster (see Rood, H. J. and Baum, W. A.: 1962, Astron. J. 73, 442) show a variation of ellipticity with radius having no obvious systematic pattern; some elliptical galaxies are also known to show rotated axes of isophotes. Could you comment on this?

Gott: In the outer envelopes of elliptical galaxies, violent relaxation is not so effective and it takes longer for the outer envelopes to reach equilibrium. Presumably, differences in initial conditions may manifest themselves as irregularities in individual cases.

Photometry of a number of elliptical galaxies by van Houten indicates that the majority of ellipticals have envelopes in which the isophotal eccentricity remains nearly constant with increasing radius. Some ellipticals, e.g. NGC 205, show rotated axes of isophotes in their outer parts which are probably due to tidal distortion (by M31 in the case of NGC 205).

G. de Vaucouleurs: Two comments: (i) the Coma cluster objects are not good tests because many are not $\mathrm{E}$, but $\mathrm{L}$ galaxies, often barred, and the high space density causes much tidal interaction. (ii) Among the best observed large $\mathrm{E}$ galaxies that are comparatively isolated, the ellipticity of the isophotes is never constant; it increases from the nucleus to a maximum which agrees roughly with the Hubble $n$ index (En), then decreases slowly in the corona (see Hubble, Astrophys. J. 71, 231, 1930; Oort, Astrophys. J. 91, 273, 1940; de Vaucouleurs, Ann. Astrophys. 11, 287, 1948; Hdb. der Phys. 53, 322, 1959; Liller, Astrophys. J. 132, 306; 1960; van Houten, Bull. Astron. Inst. Neth. 16, 1, 1961). Homothetic ellipsoids are not satisfactory models of $\mathrm{E}$ galaxies. Are these effects observed in your models?

Gott: It's hard to tell for the outermost isophotes because these regions take a long while to relax. It's not difficult to get different ellipticities. If one takes a spherical non-rotating cloud which collapses, then one expects the final galaxy to have a radius, in virial equilibrium, about half that of the initial cloud. Now by postulating differing amounts of angular momentum, the maximum being such that there is no collapse in the plane of rotation, one can get ellipticities between E0 and E5, but not significantly flatter.

G. de Vaucouleurs: I think observers would say that they've found a few E6 galaxies, such as NGC. 670, 1209, 4386, 4564, 4660, 4697, 4865, 5028, 6877 and 6909. 